\title{
Pretreatment HIV drug resistance in adults initiating antiretroviral therapy in China, 2017
}

Rui-Hua Kang ${ }^{1}$, Shu-Jia Liang ${ }^{2}$, Yan-Ling Ma ${ }^{3}$, Shu Liang ${ }^{4}$, Lin Xiao ${ }^{5}$, Xin-Hui Zhang ${ }^{6}$, Hong-Yan Lu ${ }^{7}$ Xiao-Qin Xu ${ }^{8}$, Shui-Bin Luo ${ }^{9}$, Xiao-Guang Sun ${ }^{10}$, Lin Chen ${ }^{11}$, Jian-Mei He ${ }^{12}$, Guo-Hui Wu ${ }^{13}$, Ling-Jie Liao ${ }^{1,2}$, Hui Xing ${ }^{1,2}$, Yi-Ming Shao ${ }^{1,2^{*}+}$ and Yu-Hua Ruan ${ }^{1 *+}$

\begin{abstract}
Background: After the scale-up of antiretroviral therapy (ART) for HIV infected people, increasing numbers of patients have pretreatment drug resistance (PDR). In this study, the prevalence of PDR was evaluated in adults initiating antiretroviral therapy in China.

Methods: Blood samples were obtained from 1943 patients who initiated antiretroviral therapy (ART) in 2017 from 13 provinces or cities in China. Pol sequences were used to analyze drug resistance and construct transmission networks. Logistic regression model was used to estimate the potential factors associated with PDR.

Results: In total, 1711 eligible patients (76.0\% male; 87.8\% aged $\geq 25$ years) were included, of which 117 (6.8\%) had PDR. The highest rates of PDR were 12.2\% in Liangshan Prefecture of Sichuan and 9.3 and $8.9 \%$ in Dehong and Lincang Prefecture of Yunnan. A multivariate logistic regression analysis revealed that PDR was significantly higher among intravenous drug users (adjusted Odds Ratio $(a O R)=2.64,95 \%$ Cl: 1.57-4.44) and individuals from Liangshan, Dehong, and Lincang (aOR=2.04,95\% Cl: 1.26-3.30). In total, 754 sequences were used to generate 164 transmission networks. Five transmission networks had two or three sequences containing the same mutations, two networks contained subjects from Liangshan, and one network contained subjects from Dehong.
\end{abstract}

Conclusions: Overall, the PDR prevalence was moderate, with a particularly high prevalence in areas with severe HIV epidemics. These results indicate the importance of continuous PDR monitoring in patients initiating antiretroviral therapy.

Keywords: HIV, Pretreatment drug resistance, Transmission network, Antiretroviral therapy

\footnotetext{
* Correspondence: yshao@bjmu.edu.cn; ruanyuhua92@163.com

${ }^{\dagger}$ Yi-Ming Shao and Yu-Hua Ruan contributed equally to this work.

'State Key Laboratory of Infectious Disease Prevention and Control (SKLID),

National Center for AIDS/STD Control and Prevention (NCAIDS), Chinese

Center for Disease Control and Prevention (China CDC), Collaborative

Innovation Center for Diagnosis and Treatment of Infectious Diseases, 155

Changbai Road, Changping District, Beijing, PR China

Full list of author information is available at the end of the article
}

(c) The Author(s). 2020 Open Access This article is licensed under a Creative Commons Attribution 4.0 International License, which permits use, sharing, adaptation, distribution and reproduction in any medium or format, as long as you give appropriate credit to the original author(s) and the source, provide a link to the Creative Commons licence, and indicate if changes were made. The images or other third party material in this article are included in the article's Creative Commons licence, unless indicated otherwise in a credit line to the material. If material is not included in the article's Creative Commons licence and your intended use is not permitted by statutory regulation or exceeds the permitted use, you will need to obtain permission directly from the copyright holder. To view a copy of this licence, visit http://creativecommons.org/licenses/by/4.0/. The Creative Commons Public Domain Dedication waiver (http//creativecommons.org/publicdomain/zero/1.0/) applies to the data made available in this article, unless otherwise stated in a credit line to the data. 


\section{Background}

Highly active antiretroviral therapy has decreased mortality rates and prolonged the lives of people living with HIV or AIDS (PLWHA) [1-4]. In China, the nationwide National Free Antiretroviral Treatment Program (NFATP) has been ongoing for 14 years. With the NFATP development and the formulation of the UNAIDS targets of "90-90-90" by 2020 [5], and the most important to improve the quality of life for PLWHA, the number of people receiving antiretroviral therapy (ART) has increased. With the rapid scale-up of treatment, the transmission of drug-resistant viruses can become a challenge among those newly infected with HIV. Individuals infected with drug-resistant HIV strains may experience early virological failure [6-8], and the accumulation and transmission of drug-resistant strains can result in an increased mortality rate. Pretreatment drug resistance (PDR) to standard first-line ART is a challenge for HIV treatment. The WHO recommends transmitted drug resistance surveillance among recently infected antiretroviral-naïve populations of younger than 25 years old [9]. In 2014, the WHO proposed a global strategy for the surveillance and monitoring of HIV drug resistance, including the surveillance of PDR in populations initiating ART $[10,11]$. In 2017, the WHO published new guidelines regarding the selection of antiretroviral drugs in response to high levels of PDR [12]. In particular, if the prevalence of PDR to non-nucleoside reverse-transcriptase inhibitors (NNRTIs) is $\geq 10 \%$ among individuals initiating first-line ART, excluding those with previous antiretroviral drug exposure, an alternative first-line ART regimen as a supplement should be urgently considered in accordance with the consolidated guidelines on the use of antiretroviral drugs established in 2016 [13].

In China, the NFATP was initiated at a nationwide scale in 2003 [14] and all individuals with HIV infections who provide consent can currently be treated [15]. However, genotyping was not performed for PLWHA before the initiation of ART in China. In this large crosssectional study conducted in multiple provinces in China in 2017, the prevalence of PDR among all adults was evaluated. This study is to estimate the prevalence of PDR among individuals $\geq 18$ years of age as well as the associated risk factors and to explore PDR related transmission in China.

\section{Methods}

\section{Study design and study participants}

This was a cross-sectional study to estimate the prevalence of PDR in adults initiating antiretroviral therapy in China. The method of HIVDR test was used to explore the level of PDR in part of regions in China. The study design was performed according to the 2014 WHO protocol for PDR [11]. China has twenty-three provinces, four municipalities, five autonomous regions, and two special administrative regions. HIV infection was originally identified among injected drug users (IDUs) in Yunnan Province, which is located in southwest China, and was subsequently disseminated into IDU populations in Guangxi Zhuang Autonomous Region and Sichuan Province [16-18]. HIV was subsequently disseminated by sexual transmission to populations in China. According to the numbers of newly reported HIV/AIDS in the year 2016 in China [19], the high prevalence regions were recognized when the number in the region was equal to 8000 or more; the moderate prevalence regions were defined when the number ranged from 2500 to 7999 ; and the low prevalence region refers to where the number was below 2500. This pilot investigation was conducted in high prevalence regions (Yunnan Province which included Dehong Prefecture and Lincang City, Guangxi Zhuang Autonomous Region which included Nanning City and Liuzhou City, Sichuan Province which included Liangshan Prefecture and Neijiang City, Guangdong Province which included Shenzhen City) and moderate prevalence regions (Chongqing Municipality, Guizhou Province, Hunan Province, Beijing Municipality, Jiangsu Province, and Shandong Province). WHO recommended that the number of patients would typically fall within the range of 300-500 during 6 months in a country according to the HIV drug resistance surveillance guideline in 2015 [20]. According to this guideline, at least 300 patients were recruited in high prevalence regions and moderate prevalence regions.

The patients' recruitment and specimens collection were performed from January 2017 to June 2017 (6 months). Two clinics were randomly selected in each study area. Patients were recruited at the selected clinics during the study period depending on the enrollment order. And the recruitment was based on sequential sampling of patients who received ART treatment at the clinics [20]. All recruited patients were at age 18 or older and initiated ART after this investigation. Excluded from this study were patients who were under 18 years old, or those who may be acquired drug resistance from previous antiretroviral drug exposure (such as among women exposed to ARV drugs for the prevention of mother-to-child transmission (PMTCT) of HIV, among individuals reinitiating first-line ART after a period of treatment interruption without documented viral failure or among people who have received pre-exposure prophylaxis (PrEP). All patients provided written informed consent. 


\section{Data collection}

Baseline variables were obtained for all patients, including socio-demographic and behavioural characteristics. The variables of date of birth, sex, marital status, ethnicity, years of education and route of HIV infection were achieved from the national epidemiology database of HIV infection [21]. History of ART drug use was asked by the doctor and validated by the NFATP database of HIV infection [22]. To avoid the patients with a history of ART were recruited, the doctor would reinvestigate these patients by interviewing or phone, especially these with PDR. CD4 cell counts before ART were tested within $12 \mathrm{~h}$ after sample collection and sampling region was the site of sampling clinics.

\section{Laboratory tests}

Whole blood was sampled before the initiation of ART. CD4 cells were counted by flow cytometry at local CDCs within $12 \mathrm{~h}$ of sample collection. Plasma was isolated and sent under cold-chain conditions to the laboratory at NCAIDS, China CDC. RNA was extracted from $200 \mu \mathrm{l}$ of plasma, amplified, and used to sequence the HIV pol region following an in-house method [23, 24]. The definition of any drug resistance is defined with respect to one or more of the following drugs or drug classes: Efavirenz (EFV), Nevirapine (NVP), any NRTI, Darunavir (DRV/r), Lopinavir $(\mathrm{LPV} / \mathrm{r})$, or Atazanavir (ATV/r) [11]. The classification "susceptible or potential low-level" indicates no drug resistance (Stanford penalty score $<15$ ) and a classification of at least low-level indicates drug resistance (Stanford penalty score $\geq 15$ ) [11]. PDR was evaluated using the algorithm of the Stanford HIV Drug Resistance Database (HIVDB) (https://hivdb.stanford.edu/ hivdb/by-sequences/).

\section{Identification of genetic transmission networks}

To avoid potential biases due to convergent evolution, 43 codons in $P R$ and $R T$ associated with drug resistance mutations were removed according to the most recent update of major HIV-1 drug resistance mutations [25]. Finally, the sequences were $910 \mathrm{bp}$ in length. To construct genetic transmission networks, the pairwise Tamura-Nei 93 (TN93) genetic distances $[26,27]$ were calculated among all sequences using HYPHY2.2.4. Transmission networks were visualized and analyzed using Cytoscape3.5.1 with a threshold genetic distance of 0.0125 among subtypes. The genetic distance threshold was defined as the distance that identifies the maximum number of clusters in the transmission networks [27]. The cluster included three or more same drug resistance mutations (DRMs) was defined as the HIV drug resistance (HIVDR) related cluster. The DRMs in the same clusters may be transmitted potentially. To visualize the networks, the igraph and network packages in $\mathrm{R}$ 3.5.0 software (the Free Software Foundation's GNU General Public License, Auckland, New Zealand) were used [28].

\section{Statistical analysis}

In this cross-sectional study, we collected baseline data and obtained subtypes after sequencing analysis of participants. Univariate and multivariate logistic regression models were used to estimate the potential factors associated with pretreatment drug resistance. We adjusted age, sex, marital status, ethnicity, year of education, route of infection, CD4 cell counts before ART, subtype, and region for each participant. We constructed multivariate logistic regression model in a stepwise manner to select variables independently associated with drug resistance. Odds ratio $(O R)$ and adjusted odds ratio $(\mathrm{aOR})$ with $95 \%$ confidence interval $(95 \% C I)$ were reported. All tests were two tailed and a $P$-value $<0.05$ was considered statistically significant. Statistical analyses were performed using SAS V9.4 (SAS Institute Inc., Cary, NC, USA).

\section{Results}

\section{General characteristics of the study population}

During the study period, we recruited 1943 participants in total and acquired 1731 (89.1\%) sequences. Twenty participants were excluded, including fourteen participants who were aged below 18 years and six without any information. Finally, 1711 participants were included in the study (Table 1$)$. In total, $87.8 \%(1502 / 1711)$ of participants were $\geq 25$ years old and $76.0 \%$ (1301/1711) were male. The numbers of unmarried, married, and divorced or widowed participants were 562,873 , and 271 , respectively (32.8, 51.0, and 15.8\%). Marital status was missing for five patients (0.3\%). The majority of participants $(65.9 \%, 1128 / 1711)$ were of Han ethnicity and $66.3 \%$ received a junior high school-level education or below. More than half $(60.0 \%)$ of participants were infected via heterosexual intercourse, followed by homosexual intercourse (26.5\%) and IDUs (9.4\%). In total, 1076 (62.9\%) patients had CD4 cell counts $<350$ cells $/ \mathrm{ml}$ and 576 (33.7\%) had CD4 cell counts $\geq 350$ cells $/ \mathrm{ml}$. In addition, no records of CD4 cell counts were obtained for 59 patients. The proportions of the subtypes CRF01_AE, CRF07_BC, CRF08_BC, CRF55_01B, and others were 32.7\% (559/1711), 37.0\% (633/1711), 13.3\% (228/1711), $3.7(64 / 1711)$, and $7.8 \%(134 / 1711)$, respectively. Participants were primarily from Southwest China $(52.0 \%, 889 /$ 1711). None of the participants had previous exposure to antiretroviral drugs. 
Table 1 Characteristics of HIV-infected individuals initiating ART in 2017 in China

\begin{tabular}{lll}
\hline Variable & Number & Percentage (\%) \\
\hline Total & 1711 & 100.0
\end{tabular}

Age (years)

$\begin{array}{lll}<25 & 209 & 12.2 \\ \geq 25 & 1502 & 87.8\end{array}$

Sex

Male

1301

76.0

Female

Marital status

Unmarried

562

32.8

Married

Divorce or widow

Unknown

5

Ethnic

$$
\begin{aligned}
& \text { Han } \\
& \text { Others }
\end{aligned}
$$

583

Education

Primary and below

Junior high school

Senior high school

College

Unknown

Route of infection

Heterosexual intercourse

Homosexual intercourse

Intravenous drug use

Other

CD4 count before ART (cells $/ \mathrm{mm}^{3}$ )

$$
\begin{aligned}
& <350 \\
& \geq 350
\end{aligned}
$$

Missing

Subtype

\begin{tabular}{|c|c|c|}
\hline & $N(\%)$ & $\begin{array}{l}\text { HIV drug resistance } \\
\text { mutations, } N(\%)\end{array}$ \\
\hline Total & $117(6.8)$ & \\
\hline \multirow{7}{*}{$\begin{array}{l}\text { Non-nucleoside reverse } \\
\text { transcriptase inhibitors } \\
\text { (NNRTIs, any) }\end{array}$} & $79(4.6)$ & \\
\hline & & $\mathrm{K} 103 \mathrm{~N} / \mathrm{S} / \mathrm{T}, 36(2.1)$ \\
\hline & & $\mathrm{V} 179 \mathrm{D} / \mathrm{E} / \mathrm{T}, 28(1.6)$ \\
\hline & & E138AA/K/G/Q,18 (1.1) \\
\hline & & $\mathrm{V} 106 \mathrm{M}, 9(0.5)$ \\
\hline & & G190A/S,6 (0.4) \\
\hline & & Y188L/C/F,6 (0.4) \\
\hline Efavirenz (EFV) ${ }^{a}$ & $76(4.4)$ & $\mathrm{K} 101 \mathrm{E}, 4(0.2)$ \\
\hline \multirow[t]{6}{*}{ Nevirapine (NVP) ${ }^{a}$} & $79(4.6)$ & $\mathrm{Y} 181 \mathrm{C}, 3(0.2)$ \\
\hline & & $\mathrm{H} 221 \mathrm{Y}, 3(0.2)$ \\
\hline & & A98G,2 (0.1) \\
\hline & & $\mathrm{F} 227 \mathrm{~L}, 1(0.06)$ \\
\hline & & L100I,1 (0.06) \\
\hline & & P225,1 (0.06) \\
\hline \multirow{4}{*}{$\begin{array}{l}\text { Nucleoside reverse } \\
\text { transcriptase inhibitors } \\
\text { (NRTIs, any) }\end{array}$} & $38(2.2)$ & \\
\hline & & $\mathrm{K} 65 \mathrm{R}, 9(0.5)$ \\
\hline & & $\mathrm{D} 67 \mathrm{~N}, 8(0.5)$ \\
\hline & & M184I/N,7 (0.4) \\
\hline Emtricitabine (FTC) & $12(0.7)$ & $\mathrm{T} 215 \mathrm{D} / \mathrm{I} / \mathrm{S}, 7(0.4)$ \\
\hline Lamivudine $(3 T C)^{a}$ & $12(0.7)$ & K70E/R/T,5 (0.3) \\
\hline Abacavir $(A B C)^{a}$ & $16(0.9)$ & L210W,4 (0.2) \\
\hline Didanosine $(\mathrm{DDI})^{\mathrm{a}}$ & $16(0.9)$ & $\mathrm{K} 219 \mathrm{R}, 2(0.1)$ \\
\hline Stavudine (D4T) $)^{a}$ & $32(1.9)$ & $L 74 L I, 2(0.1)$ \\
\hline Tenofovir (TDF) ${ }^{\mathrm{a}}$ & $12(0.7)$ & $M 41 L, 2(0.1)$ \\
\hline \multirow[t]{4}{*}{ Azidothymidine $(A Z T)^{a}$} & $20(1.2)$ & $\mathrm{T} 69 \mathrm{D}, 2(0.1)$ \\
\hline & & $Y 115 F, 2(0.1)$ \\
\hline & & $\mathrm{A} 62 \mathrm{~V}, 1(0.06)$ \\
\hline & & V75I,1 (0.06) \\
\hline \multirow[t]{3}{*}{ Protease inhibitors (PIs, any) } & $10(0.6)$ & \\
\hline & & $147 \mathrm{~V}, 3(0.2)$ \\
\hline & & $150 \mathrm{~V}, 2(0.1)$ \\
\hline Atazanavir (ATV) & $5(0.3)$ & $154 \mathrm{~V}, 2(0.1)$ \\
\hline Darunavir (DRV) & $4(0.2)$ & V82A/FI/L,2 (0.1) \\
\hline \multirow[t]{3}{*}{ Lopinavir (LPV) } & $10(0.6)$ & $184 \mathrm{~V}, 1(0.06)$ \\
\hline & & L90M,1 (0.06) \\
\hline & & M46I/L,1 (0.06) \\
\hline $\begin{array}{l}\text { Multi-drug resistance to NNRTI } \\
\text { and NRTI }\end{array}$ & $6(0.4)$ & \\
\hline $\begin{array}{l}\text { Multi-drug resistance to NNRTI, } \\
\text { NRTI and PI }\end{array}$ & $1(0.06)$ & \\
\hline
\end{tabular}

\begin{tabular}{lll} 
CRF01_AE & 559 & 32.7 \\
CRF07_BC & 633 & 37.0 \\
CRF08_BC & 228 & 13.3 \\
CRF55_01B & 64 & 3.7 \\
B & 40 & 2.3 \\
C & 53 & 3.1 \\
Others & 134 & 7.8 \\
\hline
\end{tabular}

Table 2 Pretreatment HIV drug resistance mutations among HIV-infected individuals with drug resistance

a National free antiviral drugs in China HIV Human immunodeficiency virus, NNRTI Non-nucleoside reverse transcriptase inhibitor, NRTI nucleoside reverse transcriptase inhibitor, PI Protease inhibitor 


\section{Pretreatment HIV drug resistance}

In total $6.8 \%(117 / 1711)$ of patients had PDR. Among them, 79 (4.6\%) had PDR to NNRTI, 38 (2.2\%) to NRTI, and $10(0.6 \%)$ to PI (Table 2). Seventy-six and seventynine participants had resistance to EFV and NVP, respectively. $K 103 N(36,2.1 \%), \operatorname{V179D}(28,1.6 \%)$, and E138A (18, $1.1 \%)$ were the most common mutations in the reverse transcriptase (RT) region. For NRTIs, the most frequent PDR drug was D4T (32, 1.9\%), followed by AZT (20, $1.2 \%)$. The most frequent mutations were $K 65 R(9,0.5 \%)$ and $D 67 N(8,0.5 \%)$ in the RT region. All 10 patients with PDR to PIs were resistant to $\mathrm{LPV} / \mathrm{r}$ and the most common mutations were $I 47 \mathrm{~V}(3,0.2 \%), I 50 \mathrm{~V}(2,0.1 \%), 154 \mathrm{~V}(2$, $0.1 \%)$, and $V 82 A / L / F(2,0.1 \%)$ in the protease (PR) region.

The prevalence of PDR varied from different regions (Table 3). The overall PDR prevalence for all regions was $6.8 \%$ (117/1711). The most severely affected drug class was NNRTI (4.6\%, 79/1711), followed by NRTI $(2.2 \%, 38 /$ $1711)$ and PI $(0.6 \%, 10 / 1711)$. Liangshan prefecture of Sichuan province had the highest PDR prevalence $(12.2 \%$, $34 / 279$ ) among all regions, followed by Dehong prefecture $(9.3 \%, 14 / 150)$ and Lincang prefecture $(8.9 \%, 14 / 158)$ of Yunnan province.

\section{Factors associated with HIV PDR}

Risk factors associated with HIV PDR are listed in Table 4. In a univariate logistic regression analysis, four factors were significantly associated with HIV PDR. The $O R$ for patients infected via IDU versus heterosexual intercourse was 3.61 (95\% CI: 2.22-
5.88) and that for Liangshan, Dehong, and Lincang versus other regions was 2.30 (95\% CI: 1.57-3.35). A multivariate logistic regression model showed that IDUs and region (Liangshan, Dehong, and Lincang) were important factors, with aORs of 2.64 (95\% CI: $1.57-4.44)$ and 2.04 (95\% CI: 1.26-3.30), respectively.

\section{Genetic transmission networks}

In total, $1711 \mathrm{pol}$ sequences were obtained to construct genetic transmission networks. We constructed transmission networks with a genetic distance threshold of 0.0125 , which identified the maximum number of clusters. In total, 754 (44.1\%) sequences were used to generate 164 transmission networks (Fig. 1). Among all transmission networks, $70.1 \%$ (115 of 164) included 2 individuals.

Overall, 43 sequences with PDR were included in the networks and the degrees between PDR and no PDR in the networks were not statistically significant. The 43 sequences with PDR were dispersed among 23 transmission networks; shared mutations yielded five transmission networks (Fig. 1). The most frequent DRMs in the transmission networks was K103N $(7.9 \%, 13 / 164)$. Three transmission networks contained two PDR sequences with the K103N mutation and they were from Liangshan prefecture of Sichuan province, Dehong Prefecture of Yunnan Province, and Liuzhou City of Guangxi Province. In addition, three PDR sequences with E138Q and V179D mutations comprised a transmission network and they were from

Table 3 Pretreatment HIV drug resistance among HIV-infected individuals initiating ART in 2017 in China, by region

\begin{tabular}{|c|c|c|c|c|c|c|}
\hline & \multirow[t]{2}{*}{ Number } & \multirow{2}{*}{$\begin{array}{l}\text { Number } \\
\text { of PDR }\end{array}$} & \multirow{2}{*}{$\begin{array}{l}\text { Prevalence } \\
(\%)\end{array}$} & \multicolumn{3}{|c|}{ Prevalence (\%) } \\
\hline & & & & NNRTIS & NRTIS & $\mathrm{Pls}$ \\
\hline Total & 1711 & 117 & 6.8 & $4.6(79 / 1711)$ & $2.2(38 / 1711)$ & $0.6(10 / 1711)$ \\
\hline High prevalence regions & 1102 & 90 & 8.2 & $5.5(61 / 1102)$ & $2.8(31 / 1102)$ & $0.6(7 / / 1102)$ \\
\hline Dehong, Yunnan & 150 & 14 & 9.3 & $8.7(13 / 150)$ & $0.7(1 / 150)$ & 0 \\
\hline Lincang, Yunnan & 158 & 14 & 8.9 & $5.7(9 / 158)$ & $3.8(6 / 158)$ & $1.3(2 / 158)$ \\
\hline Liuzhou, Guangxi & 207 & 11 & 5.3 & $2.9(6 / 207)$ & $1.9(4 / 207)$ & $0.5(1 / 207)$ \\
\hline Nanning, Guangxi & 104 & 7 & 6.7 & $3.9(4 / 104)$ & $3.8(4 / 104)$ & 0 \\
\hline Liangshan, Sichuan & 279 & 34 & 12.2 & $9.0(25 / 279)$ & $3.9(11 / 279)$ & $1.1(3 / 279)$ \\
\hline Neijiang, Sichuan & 105 & 5 & 4.8 & $1.0(1 / 105)$ & $2.9(3 / 105)$ & $1.0(1 / 105)$ \\
\hline Shenzhen, Guangdong & 99 & 5 & 5.1 & $3.0(3 / 99)$ & $2.0(2 / 99)$ & 0 \\
\hline Moderate prevalence regions & 609 & 27 & 4.4 & $3.0(18 / 609)$ & $1.1(7 / 609)$ & $0.5(3 / 609)$ \\
\hline Chongqing & 85 & 5 & 5.9 & $2.4(2 / 85)$ & $1.2(1 / 85)$ & $2.4(2 / 85)$ \\
\hline Guizhou & 112 & 5 & 4.5 & $1.8(2 / 112)$ & $1.8(2 / 112)$ & $0.9(1 / 112)$ \\
\hline Hunan & 97 & 2 & 2.1 & $2.1(2 / 97)$ & $1.0(1 / 97)$ & 0 \\
\hline Beijing & 111 & 4 & 3.6 & $2.7(3 / 111)$ & $0.9(1 / 111)$ & 0 \\
\hline Jiangsu & 105 & 5 & 4.8 & $4.8(5 / 105)$ & 0 & 0 \\
\hline Shandong & 99 & 6 & 6.1 & $4.0(4 / 99)$ & $2.0(2 / 99)$ & 0 \\
\hline
\end{tabular}

PDR Pretreatment drug resistance, NNRTI Non-nucleoside reverse transcriptase inhibitor, NRTI nucleoside reverse transcriptase inhibitor, PI Protease inhibitor. 
Table 4 Factors associated with pretreatment HIV drug resistance among HIV-infected individuals initiating ART in 2017 in China

\begin{tabular}{|c|c|c|c|c|c|c|}
\hline Variable & Number & PDR, N (\%) & OR $(95 \% C l)$ & $P$-value & $\mathrm{aOR}(95 \% \mathrm{Cl})$ & $P$-value \\
\hline Total & 1711 & $117(6.8)$ & & & & \\
\hline \multicolumn{7}{|l|}{ Age (years) } \\
\hline$<25$ & 209 & $16(7.7)$ & 1.00 & & & \\
\hline$\geq 25$ & 1502 & $101(6.7)$ & $0.87(0.50-1.51)$ & 0.62 & & \\
\hline \multicolumn{7}{|l|}{ Sex } \\
\hline Male & 1301 & $93(7.2)$ & 1.00 & & & \\
\hline Female & 410 & $24(5.9)$ & $0.76(0.48-1.22)$ & 0.26 & & \\
\hline \multicolumn{7}{|l|}{ Marital status } \\
\hline Unmarried & 562 & $38(6.8)$ & 1.00 & & & \\
\hline Married & 873 & $59(6.8)$ & $1.00(0.66-1.53)$ & 0.99 & & \\
\hline Divorce or widow & 271 & $20(7.4)$ & $1.10(0.63-1.93)$ & 0.74 & & \\
\hline Unknown & 5 & $0(0.0)$ & - & 0.99 & & \\
\hline \multicolumn{7}{|l|}{ Education } \\
\hline Primary and below & 638 & $52(8.2)$ & 1.00 & & & \\
\hline Junior high school & 496 & $35(7.1)$ & $0.86(0.55-1.34)$ & 0.49 & & \\
\hline Senior high school & 217 & $14(6.5)$ & $0.78(0.42-1.43)$ & 0.42 & & \\
\hline College & 307 & $15(4.9)$ & $0.58(0.32-1.05)$ & 0.07 & & \\
\hline Unknown & 53 & $1(1.9)$ & $0.22(0.03-1.60)$ & 0.13 & & \\
\hline \multicolumn{7}{|l|}{ Route of infection } \\
\hline Heterosexual intercourse & 1027 & $57(5.6)$ & 1.00 & & 1.00 & \\
\hline Homosexual intercourse & 453 & $27(6.0)$ & $1.08(0.67-1.73)$ & 0.75 & $1.48(0.87-2.52)$ & 0.15 \\
\hline Intravenous drug use & 160 & $28(17.5)$ & $3.61(2.22-5.88)$ & $<0.001$ & $2.64(1.57-4.44)$ & $<0.001$ \\
\hline Others & 71 & $5(7.0)$ & $1.29(0.50-3.33)$ & 0.60 & $1.56(0.60-4.08)$ & 0.36 \\
\hline \multicolumn{7}{|l|}{ CD4 count before ART (cells/mm³) } \\
\hline$<350$ & 1076 & $71(6.6)$ & 1.00 & & & \\
\hline$\geq 350$ & 576 & $45(7.8)$ & $1.20(0.81-1.77)$ & 0.36 & & \\
\hline Missing & 59 & $1(1.7)$ & $0.24(0.03-1.79)$ & 0.17 & & \\
\hline \multicolumn{7}{|l|}{ Subtype } \\
\hline CRF01_AE & 559 & $26(4.7)$ & 1.00 & & & \\
\hline CRF07_BC & 633 & $49(7.7)$ & $1.72(1.05-2.81)$ & 0.03 & & \\
\hline CRF08_BC & 228 & $19(8.3)$ & $1.86(1.01-3.44)$ & 0.05 & & \\
\hline CRF55_01B & 64 & $8(12.5)$ & $2.93(1.27-6.78)$ & 0.01 & & \\
\hline B & 40 & $1(2.5)$ & $0.53(0.07-3.98)$ & 0.53 & & \\
\hline C & 53 & $5(9.4)$ & $2.14(0.78-5.81)$ & 0.14 & & \\
\hline Others & 134 & $9(6.7)$ & $1.48(0.68-3.23)$ & 0.33 & & \\
\hline \multicolumn{7}{|l|}{ Region } \\
\hline Others & 1124 & $55(4.9)$ & 1.00 & & 1.00 & \\
\hline Liangshan, Dehong and Lincang ${ }^{a}$ & 587 & $62(10.6)$ & $2.30(1.57-3.35)$ & $<0.001$ & $2.04(1.26-3.30)$ & 0.004 \\
\hline
\end{tabular}

${ }^{a}:$ The HIV epidemic was the most severe in Liangshan, Dehong and Lincang. The HIVDR rate was high in these areas

$P D R$ pretreatment drug resistance, $O R$ odds ratio, aOR adjusted odds ratio, $C l$ confidence interval, $A R T$ antiretroviral therapy, HIV Human immunodeficiency virus, HIVDR HIV drug resistance; -: Not applicable.

Liangshan prefecture of Sichuan province. Another transmission network included two PDR sequences with the L210W mutation from Shandong Province.
Among the 754 individuals included in the transmission networks, 49 were infected via IDU. Among these 49 individuals, five had PDR in five different networks. 


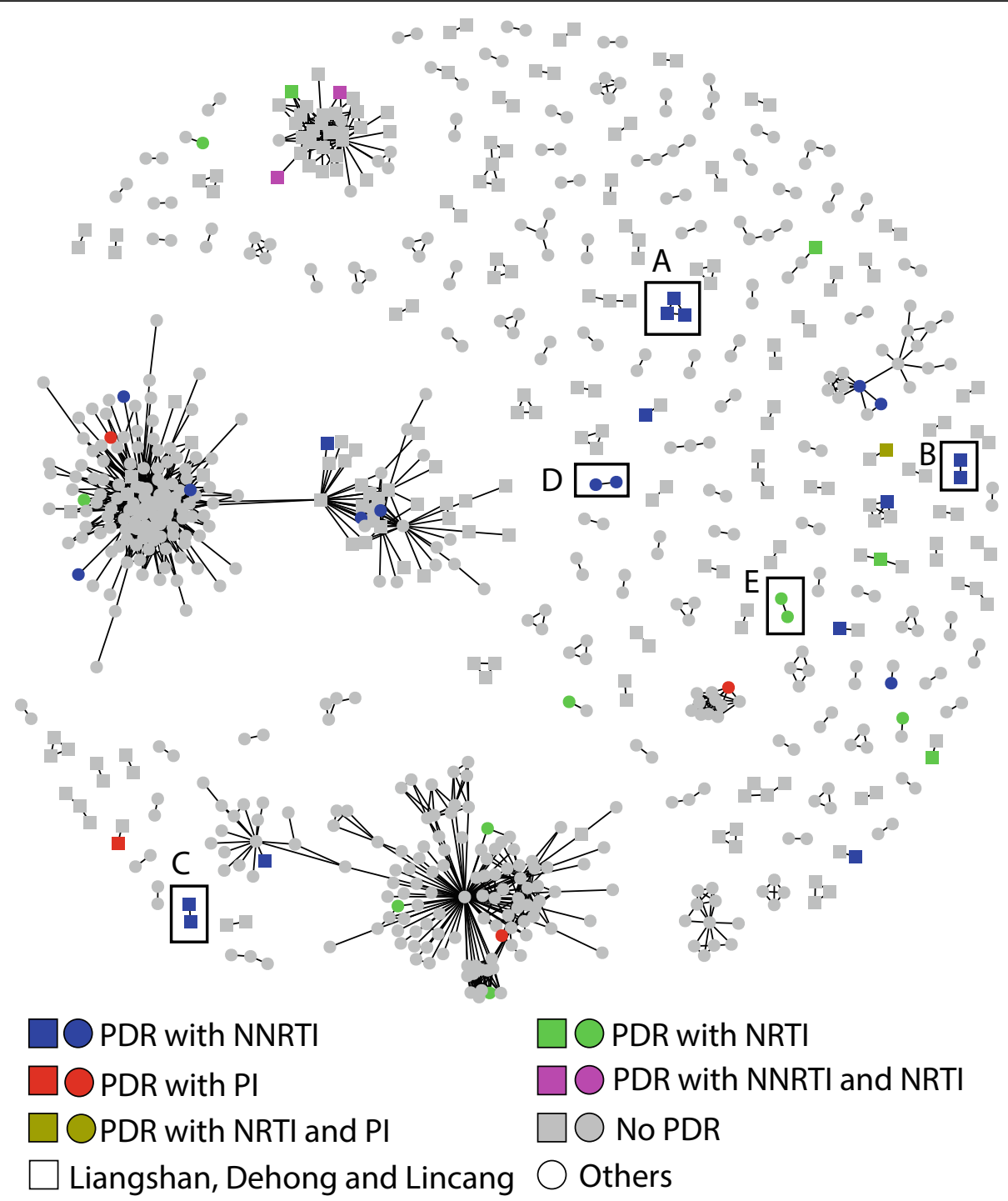

Fig. 1 Pretreatment HIV drug resistance related transmission networks. Note: a Three sequences were from Liangshan and drug resistance mutations were $E 138 \mathrm{Q}$ and V179D. b Two sequences were from Liangshan and drug resistance mutation was K103N. c Two sequences were from Dehong and drug resistance mutations were K103N and V106M. d Two sequences were from Liuzou and drug resistance mutation was K103N. e Two sequences were from Shandong and drug resistance mutation was L210W. PDR: pretreatment drug resistance; NNRTI: non-nucleoside reverse-transcriptase inhibitor; NRTI: Nucleoside reverse-transcriptase inhibitor; PI: Protease inhibitor

No transmission of the virus with PDR was observed among individuals who inject drugs.

\section{Discussion}

We performed a cross-sectional study of HIV PDR in China and obtained an overall PDR prevalence of $6.8 \%$ (95\% CI: 6.5-7.1\%). According to the WHO, low, moderate, and high levels of HIV drug resistance are defined as $<5 \%$, $5-15 \%$, and $>15 \%$ [10]. PDR prevalence was moderate in China in 2017. The pooled prevalence of PDR was similar or higher than those for other countries, such as Zimbabwe (6.3\%) [29] and Sub-Saharan Africa (5.5\%) [30] but lower than estimates for Kenya (9.4\%) [27], Argentina (14.0\%) [31], and Mexico (15.5\%) [32]. The prevalence of NNRTI PDR was $4.6 \%$ (95\% CI: 4.4-4.8\%) in this study, which is below the $10 \%$ threshold to change the recommended firstline ART regimen [12]. However, six countries have reported rates of PDR to NNRTI of $>10 \%$ [33]. Studies have suggested that PDR is associated with a shorter time to virological failure or increased risk of ART regimen switch $[7,30,32]$. Moreover, the most common mutation in this study was K103N, consistent with studies in Argentina, Zimbabwe, and Kenya [29, 31, 34]. Hence, routine surveillance programs for PDR in China are necessary. 
In this study, there were no associations between age, sex, or CD4 cell counts before ART and PDR. These results were consistent with previous results in Vietnam and Zimbabwe [29, 35]. However, IDU was significantly associated with PDR, as observed in a previous study in Vietnam [29]. Another study has shown that IDUs receiving first-line ART have a higher rate of virological failure in China [36]. Although the overall prevalence of PDR in our study was moderate, the prevalence was high in some regions, i.e., Liangshan Prefecture of Sichuan, in Dehong Prefecture, and Lincang of Yunnan. Liangshan prefecture of Sichuan and Dehong prefecture of Yunnan are areas with serious HIV epidemics and IDUs are the main highrisk population for HIV infection. Antiretroviral treatment was initiated early and the highest rates of HIV-infected people receiving ART are in Sichuan and Yunnan.

In China, the recommended first-line regimen was zidovudine (AZT) or stavudine (D4T) + lamivudine (3TC) + nevirapine (NVP) in 2005 [22] and D4T was gradually replaced by AZT or tenofovir (TDF) from 2010 [37]. The current first-line regimen was TDF or AZT + 3TC + efavirenz (EFV) or NVP [15]. This study indicated that the HIVDR was high in the high prevalence regions, even though these patients without antiretroviral drugs exposure. The most common DRMs were related these drugs being used in China. Hence, the DRMs in treatment-naïve patients may originate from treatment failure.

Transmission networks were constructed to explore whether the HIVDR related cluster was existed among PLWHA before the initiation of ART. There were no significant differences between the degrees of PDR and no PDR. However, there were five transmission networks with two or three same DRMs. Two transmission networks contained subjects from Liangshan, and one contained subjects from Dehong. Another contained subjects from Liuzhou. All these cities are in regions with the most severe HIV epidemics. One additional transmission network contained subjects from Shandong, with moderate HIV rates. There was a HIVDR related cluster contained three same DRMs in Liangshan, especially. These results suggest that PDR could be transmitted among high-risk populations for HIV infection in the future.

The study had the following limitations. First, China has seven regions (northeast, north, northwest, central, east, south, and southwest). This pilot study survey did not include all these regions and was not representative of all individuals living with HIV or AIDS in China. We will expand the PDR study to all provinces in inland China next year. Second, the lack of drug-resistant virus transmission among IDUs in this study could be related to the sampling density. IDUs were not infected in local areas or related individuals were not recruited in this study.

\section{Conclusions}

The present results demonstrate that the overall prevalence of PDR is moderate in China. However, in Liangshan prefecture of Sichuan, it exceeds 10\%. There was no ongoing transmission of resistance-associated mutations in the networks. However, regional differences and complex epidemic trends suggest that the continuous surveillance of PDR is essential for estimating the level of PDR and ensuring the effectiveness of current ART regimens in China.

\section{Abbreviations \\ HIV: Human immunodeficiency virus; PDR: Pretreatment drug resistance; PLWHA: People living with HIV or AIDS; UNAIDS: United Nations programme on HIV/AIDS; WHO: World Health Organization; ART: Antiretroviral therapy; ARV: Antiretroviral virus; NFATP: National Free Antiretroviral Treatment Program; NNRTI: Non-nucleoside reverse-transcriptase inhibitor; NRTI: Nucleoside reverse-transcriptase inhibitor; PI: Protease inhibitor; IDUs: Injected drug users; PMTCT: Prevention of mother-to-child transmission; PrEP: Pre-exposure prophylaxis; EFV: Efavirenz; NVP: Nevirapine; FTC: Emtricitabine; 3TC: Lamivudine; ABC: Abacavir; DDI: Didanosine; D4T: Stavudine; TDF: Tenofovir; AZT: Azidothymidine; DRV/r: Darunavir; LPV/r : Lopinavir; ATV/r: Atazanavir; DRM: Drug resistance mutation; HIVDR: HIV Drug Resistance Database; TN93: Tamura-Nei 93; OR: Odds ratio; aOR: Adjusted odds ratio; Cl: Confidence interval}

\section{Acknowledgments}

Data in this manuscript were collected by the Beijing, Jiangsu, Shandong, Hunan, Shenzhen, Guangxi, Neijiang, Liangshan, Chongqing, Guizhou, and Yunnan Center for Disease Control and Prevention.

\section{Authors' contributions}

RK, LL, HX, YS and YR were responsible for study design and planning. RK, SL, $Y M, S L, L X, X Z, H L, X X, S L, X S, L C, J H, G W, L L, H X, Y S$ and YR contributed to data collection. RK, LL, HX, YS, YR contributed to data analysis and interpretation. RK, LL, HX, YS, YR contributed to writing the manuscript. All authors read and approved the final version of the manuscript.

\section{Funding}

This study was supported by the National Natural Science Foundation of China (11471336), Ministry of Science and Technology of China

(2017ZX10201101), Guangxi Science and Technology Bureau (AB16380213), Guangxi Bagui Honor Scholarship, and Chinese State Key Laboratory of Infectious Disease Prevention and Control.

\section{Availability of data and materials}

The datasets are available from the corresponding author on reasonable request.

\section{Ethics approval and consent to participate}

Institutional review board approval was granted by the National Center for AIDS/STD Control and Prevention (NCAIDS), Chinese Center for Disease Control and Prevention (China CDC).

\section{Consent for publication}

Not applicable.

\section{Competing interests}

The authors declare that they have no competing interests.

\section{Author details}

${ }^{1}$ State Key Laboratory of Infectious Disease Prevention and Control (SKLID), National Center for AIDS/STD Control and Prevention (NCAIDS), Chinese Center for Disease Control and Prevention (China CDC), Collaborative Innovation Center for Diagnosis and Treatment of Infectious Diseases, 155 Changbai Road, Changping District, Beijing, PR China. ${ }^{2}$ Guangxi Center for Disease Control and Prevention, Nanning, Guangxi, China. ${ }^{3}$ Yunnan Center for Disease Control and Prevention, Kunming, Yunnan, China. ${ }^{4}$ Sichuan Center for Disease Control and Prevention, Chengdu, Sichuan, China.

${ }^{5}$ Liangshan Center for Disease Control and Prevention, Liangshan prefecture, 
Sichuan, China. ${ }^{6}$ Guizhou Center for Disease Control and Prevention, Guiyang, Guizhou, China. 'Beijing Center for Disease Control and Prevention, Beijing, China. ${ }^{8}$ Jiangsu Center for Disease Control and Prevention, Nanjing, Jiangsu, China. ${ }^{9}$ Neijiang Center for Disease Control and Prevention, Neijiang, Sichuan, China. ${ }^{10}$ Shandong Center for Disease Control and Prevention, Jinan, Shandong, China. " ${ }^{1}$ Shenzhen Center for Disease Control and Prevention, Shenzhen, Guangdong, China. ${ }^{12}$ Hunan Center for Disease Control and Prevention, Changsha, Hunan, China. ${ }^{13}$ Chongqing Center for Disease Control and Prevention, Chongqing, China.

Received: 13 November 2019 Accepted: 6 May 2020

Published online: 24 May 2020

\section{References}

1. Lima VD, Lourenço L, Yip B, Hogg RS, Phillips P, Montaner JS. AIDS incidence and AIDS-related mortality in British Columbia, Canada, between 1981 and 2013: a retrospective study. Lancet HIV. 2015;2(3):e92-7.

2. Ford N, Kranzer K, Hilderbrand K, Jouquet G, Goemaere E, Vlahakis N, et al. Early initiation of antiretroviral therapy and associated reduction in mortality, morbidity and defaulting in a nurse-managed, community cohort in Lesotho. AIDS. 2010;24(17):2645-50.

3. Johnson LF, Mossong J, Dorrington RE, Schomaker M, Hoffmann CJ, Keiser O, et al. Life expectancies of south African adults starting antiretroviral treatment: collaborative analysis of cohort studies. PLoS Med. 2013;10(4):e1001418.

4. Antiretroviral Therapy Cohort Collaboration. Life expectancy of individuals on combination antiretroviral therapy in high-income countries: a collaborative analysis of 14 cohort studies. Lancet. 2008;372(9635):293-9.

5. Title of subordinate document. In: 90-90-90 An ambitious treatment target to help end the AIDS epidemic. UNAIDS. 2014. http://www.unaids.org/sites/ default/files/media_asset/90-90-90_en.pdf. Accessed 3 Nov 2019.

6. Hamers RL, Schuurman R, Sigaloff KC, Wallis CL, Kityo C, Siwale M, et al. Effect of pretreatment HIV-1 drug resistance on immunological, virological, and drug resistance outcomes of first-line antiretroviral treatment in sub-Saharan Africa: a multicentre cohort study. Lancet Infect Dis. 2012;12(4):307-17.

7. Kantor R, Smeaton L, Vardhanabhuti S, Hudelson SE, Wallis CL, Tripathy S, et al. Pretreatment HIV drug resistance and HIV-1 subtype $C$ are independently associated with virologic failure: results from the multinational PEARLS (ACTG A5175) clinical trial. Clin Infect Dis. 2015;60(10): 1541-9.

8. Kityo C, Boerma RS, Sigaloff KC, Kaudha E, Calis JCJ, Musiime V, et al Pretreatment HIV drug resistance results in virological failure and accumulation of additional resistance mutations in Ugandan children. J Antimicrob Chemother. 2017;72(9):2587-95.

9. Bennett DE, Myatt M, Bertagnolio S, Sutherland D, Gilks CF. Recommendations for surveillance of transmitted HIV drug resistance in countries scaling up antiretroviral treatment. Antivir Ther. 2008;13(Suppl 2):25-36.

10. Title of subordinate document. In: World Health Organization global strategy for the surveillance and monitoring of HIV drug resistance. WHO. 2012 www.who.int/hiv/pub/drugresistance/drug_resistance_strategy/en/. Accessed 3 Nov 2019.

11. Title of subordinate document. In: Surveillance of HIV drug resistance in adults initiating antiretroviral therapy (pre-treatment HIV drug resistance). WHO. 2014. www.who.int/hiv/pub/drugresistance/pretreatment_ drugresistance/en/. Accessed 3 Nov 2019.

12. Title of subordinate document. In: Guidlines on the public health response to pretreatment HIV drug resistance. WHO. 2017. www.who.int/hiv/pub/ guidelines/hivdr-guidelines-2017/en/. Accessed 3 Nov 2019.

13. Title of subordinate document. In: Consolidated guidelines on the use of antiretroviral drugs for treating and preventing HIV infection. WHO. 2016. www.who.int/news-room/fact-sheets/detail/hiv-aids. Accessed 3 Nov 2019.

14. Zhang F, Haberer JE, Wang Y, Zhao Y, Ma Y, Zhao D, et al. The Chinese free antiretroviral treatment program: challenges and responses. AIDS. 2007; 21(Suppl 8):S143-8.

15. National center for AIDS/STD Control and Prevention. Manual of the National Free Antiretroviral Treatment, 4th ed. China: People's Medical Publishing House; 2016. p. 14

16. Luo CC, Tian C, Hu DJ, Kai M, Dondero T, Zheng X. HIV-1 subtype C in China. Lancet. 1995;345(8956):1051-2.

17. Li Z, He X, Wang Z, Xing H, Li F, Yang Y, et al. Tracing the origin and history of HIV-1 subtype B' epidemic by near full-length genome analyses. AIDS. 2012;26(7):877-84
18. Piyasirisilp S, McCutchan FE, Carr JK, Sanders-Buell E, Liu W, Chen J, et al. A recent outbreak of human immunodeficiency virus type 1 infection in southern China was initiated by two highly homogeneous, geographically separated strains, circulating recombinant form $A E$ and a novel $B C$ recombinant. J Virol. 2000;74(23):11286-95.

19. National center for AIDS/STD Control and Prevention, China CDC. Annual Report on Provincial AIDS/STD Surveillance in 2016.

20. Title of subordinate document. In: HIV drug resistance surveillance guideline: 2015 update. https://apps.who.int/iris/handle/10665/204471. Accessed 5 Feb 2020.

21. Zhang FJ, Dou ZH, Ma Y, Zhang Y, Zhao Y, Zhao DC, et al. Effect of earlier initiation of antiretroviral treatment and increased treatment coverage on HIV-related mortality in China: a national observational cohort study. Lancet Infect Dis. 2011;11:516-24.

22. Title of subordinate document. In: Manual of the National Free Antiretrovira Treatment, first edition. 2005. http://www.ncaids.chinacdc.cn/ls/m/200501/t2 0050131_1075189.html. Accessed 16 Sep 2016.

23. Liao L, Xing H, Shang H, Li J, Zhong P, Kang L, et al. The prevalence of transmitted antiretroviral drug resistance in treatment-naive HIV-infected individuals in China. J Acquir Immune Defic Syndr. 2010;53(Suppl 1):S10-4

24. Xing H, Ruan Y, Hsi JH, Kan W, Liao L, Leng $X$, et al. Reductions in virological failure and drug resistance in Chinese antiretroviral-treated patients due to lamivudine-based regimens 2003-12. J Antimicrob Chemother. 2015;70(7):2097-103.

25. Title of subordinate document. In: Major HIV-1 drug resistance mutations. 2017. https://hivdb.stanford.edu/. Accessed 3 Nov 2019.

26. Tamura K, Nei M. Estimation of the number of nucleotide substitutions in the control region of mitochondrial DNA in humans and chimpanzees. Mol Biol Evol. 1993;10(3):512-26.

27. Wertheim JO, Kosakovsky Pond SL, Forgione LA, Mehta SR, Murrell B, Shah $S$, et al. Social and genetic networks of HIV-1 transmission in New York City. PLoS Pathog. 2017;13(1):e1006000.

28. Butts C. T: network: a package for managing relational data in R. J Stat Softw. 2008:24(2):36.

29. Mungati M, Mhangara M, Gonese E, Mugurungi O, Dzangare J, Ngwende $S$, et al. Pre-treatment drug resistance among patients initiating antiretroviral therapy (ART) in Zimbabwe: 2008-2010. BMC Res Notes. 2016:9:302

30. Boender TS, Hoenderboom BM, Sigaloff KC, Hamers RL, Wellington M, Shamu T, et al. Pretreatment HIV drug resistance increases regimen switches in sub-Saharan Africa. Clin Infect Dis. 2015;61(11):1749-58

31. Bissio E, Barbas MG, Bouzas MB, Cudolá A, Salomón H, Espínola, et al. Pretreatment HIV-1 drug resistance in Argentina: results from a surveillance study performed according to WHO-proposed new methodology in 201415. J Antimicrob Chemother. 2017:72(2):504-10.

32. Avila-Rios S, Garcia-Morales C, Matias-Florentino M, Romero-Mora KA, TapiaTrejo D, Quiroz-Morales VS, et al. Pretreatment HIV-drug resistance in Mexico and its impact on the effectiveness of first-line antiretroviral therapy: a nationally representative 2015 WHO survey. Lancet HIV. 2016;3(12):e579-91.

33. Title of subordinate document. In: HIV Drug Resistance Report 2017. WHO. 2017. www.who.int/hiv/pub/drugresistance/hivdr-report-2017/en/. Accessed 3 Nov 2019.

34. Silverman RA, Beck IA, Kiptinness C, Levine M, Milne R, McGrath CJ, et al. Prevalence of pre-antiretroviral-treatment drug resistance by gender age and other factors in HIV-infected individuals initiating therapy in Kenya, 2013-2014. J Infect Dis. 2017:216(12):1569-78.

35. Pham QD, Do NT, Le YN, Nguyen TV, Nguyen DB, Huynh TK, et al. Pretreatment HIV-1 drug resistance to first-line drugs: results from a baseline assessment of a large cohort initiating ART in Vietnam 2009-10. J Antimicrob Chemother. 2015:70(3):941-7.

36. Leng X, Liang S, Ma Y, Dong Y, Kan W, Goan D, et al. HIV virological failure and drug resistance among injecting drug users receiving first-line ART in China. BMJ Open. 2014;4(10):e005886.

37. Book writing group. Manual of the National Free Antiretroviral Treatment. 2nd ed. Beijing: People's Medical Publishing House; 2008. 